\title{
Disorder-specific grey matter deficits in attention deficit hyperactivity disorder relative to autism spectrum disorder
}

\author{
L. Lim $^{1,2 *}$, K. Chantiluke ${ }^{1}$, A. I. Cubillo ${ }^{1}$, A. B. Smith ${ }^{1}$, A. Simmons ${ }^{3,4}$, M. A. Mehta ${ }^{3}$ and K. Rubia ${ }^{1}$ \\ ${ }^{1}$ Department of Child and Adolescent Psychiatry, Institute of Psychiatry, King's College London, UK \\ ${ }^{2}$ Department of Psychological Medicine, Yong Loo Lin School of Medicine, National University of Singapore, Singapore \\ ${ }^{3}$ Department of Neuroimaging, Institute of Psychiatry, King's College London, UK \\ ${ }^{4}$ NIHR Biomedical Research Centre at South London and Maudsley NHS Foundation Trust (SLaM), London, UK
}

Background. Attention deficit hyperactivity disorder (ADHD) and autism spectrum disorder (ASD) are two common childhood disorders that exhibit genetic and behavioural overlap and have abnormalities in similar brain systems, in particular in frontal and cerebellar regions. This study compared the two neurodevelopmental disorders to investigate shared and disorder-specific structural brain abnormalities.

Method. Forty-four predominantly medication-naïve male adolescents with ADHD, 19 medication-naïve male adolescents with ASD and 33 age-matched healthy male controls were scanned using high-resolution T1-weighted volumetric imaging in a 3-T magnetic resonance imaging (MRI) scanner. Voxel-based morphometry (VBM) was used to test for group-level differences in structural grey matter (GM) and white matter (WM) volumes.

Results. There was a significant group difference in the GM of the right posterior cerebellum and left middle/superior temporal gyrus (MTG/STG). Post-hoc analyses revealed that this was due to ADHD boys having a significantly smaller right posterior cerebellar GM volume compared to healthy controls and ASD boys, who did not differ from each other. ASD boys had a larger left MTG/STG GM volume relative to healthy controls and at a more lenient threshold relative to ADHD boys.

Conclusions. The study shows for the first time that the GM reduction in the cerebellum in ADHD is disorder specific relative to ASD whereas GM enlargement in the MTG/STG in ASD may be disorder specific relative to ADHD. This study is a first step towards elucidating disorder-specific structural biomarkers for these two related childhood disorders.

Received 17 December 2013; Revised 20 July 2014; Accepted 21 July 2014; First published online 17 September 2014

Key words: ADHD, ASD, cerebellum, magnetic resonance imaging, superior temporal lobe, voxel-based morphometry.

\section{Introduction}

Attention deficit hyperactivity disorder (ADHD) is one of the most commonly diagnosed childhood disorders, defined by age-inappropriate problems with inattention, impulsivity and hyperactivity (APA, 2013). Autism spectrum disorder (ASD) is characterized by abnormalities in social interaction, communication and stereotyped/repetitive behaviours. Both disorders are highly heritable and share high comorbidity (Simonoff et al. 2008; Rommelse et al. 2010). About $20-50 \%$ of ADHD children meet criteria for ASD and $30-80 \%$ of ASD children meet criteria for ADHD (Rommelse et al. 2010). Apart from comorbidities, ASD patients show some ADHD-typical behaviours

\footnotetext{
* Address for correspondence: L. Lim, Department of Child and Adolescent Psychiatry, Institute of Psychiatry, King's College London, London SE5 8AF, UK.

(Email: lena.lim@kcl.ac.uk)
}

such as attention deficits, impulsivity or hyperactivity (Schatz et al. 2002) whereas ADHD patients also show some social interaction and communication difficulties, albeit to a smaller degree than ASD patients (Geurts et al. 2004).

Despite the reported genetic and behavioural overlap between the two disorders, a diagnosis of ADHD according to DSM-IV (APA, 2000) and ICD-10 (WHO, 1994) was precluded if the symptoms were better accounted for by autism. It has been debated whether the phenotypically similar ADHD-related deficits in ASD are secondary to ASD or a phenocopy, which had prevented the co-diagnosis in DSM-IV and ICD-10 (APA, 2000), or whether they reflect true comorbidity, as suggested in the allowance for co-diagnosis in the current DSM-5 criteria (APA, 2013). The identification of both overlapping and disorder-specific objective neurobiological biomarkers should help to determine to what extent the two disorders differ in their underlying neurobiology. 
ADHD is a multi-systemic neurodevelopmental disorder that has consistently been associated with abnormalities in structure, function and inter-regional connectivity of fronto-striato-parieto-temporal and fronto-cerebellar networks (Valera et al. 2007; Nakao et al. 2011; Rubia, 2011; Cubillo et al. 2012a; Rubia et al. 2014). Structural magnetic resonance imaging (sMRI) studies using region of interest (ROI) analyses have reported reduced grey matter (GM) volume and cortical thickness, most prominently in the cerebellar hemispheres (Castellanos et al. 2002; Durston et al. 2004; Mackie et al. 2007; Valera et al. 2007) and cerebellar vermis (Mackie et al. 2007; Valera et al. 2007), but also in the basal ganglia and frontal regions (Shaw et al. 2007). Whole-brain sMRI studies, however, found that the most consistent GM reductions were in the basal ganglia (Nakao et al. 2011; Frodl \& Skokauskas, 2012).

sMRI studies have reported abnormal GM volumes and cortical thickness in ASD patients relative to controls in several brain regions involved in social, language and executive functions, including prefrontal, temporo-parietal, striatal, limbic and cerebellar regions (Amaral et al. 2008; Nickl-Jockschat et al. 2012). However, findings have been inconsistent with respect to the direction of GM differences; some studies found increases in GM volumes or cortical thickness (Bonilha et al. 2008; Hyde et al. 2010) whereas others found decreases (Brun et al. 2009; Webb et al. 2009; Toal et al. 2010) or no differences (Hazlett et al. 2005; Scott et al. 2009). Studies on the developmental course of brain abnormalities in autism indicate a putative period of abnormal precocious brain growth that is time delimited to the first 2-4 years of life but then plateaus by adolescence and adulthood (Amaral et al. 2008; Courchesne et al. 2011), with some studies finding arrested growth after adolescence (Amaral et al. 2008).

Despite evidence for high comorbidity rates and abnormalities in similar brain systems, in particular in frontal and cerebellar regions, few studies have compared the two disorders to elucidate shared and disorder-specific underlying neurobiological biomarkers. The only sMRI study to date that compared relatively small numbers of 15 ADHD and 15 ASD children found shared reductions in the GM of temporoparietal regions and also increased GM of the supramarginal gyrus in ASD relative to controls, but not ADHD (Brieber et al. 2007). However, the findings did not survive correction for multiple testing. In addition, most ADHD patients were on chronic stimulant medication and two ASD patients took neuroleptic medication that could have confounded the findings, given that long-term psychotropic medication is associated with more normal brain structure (Shaw et al. 2009; Murphy, 2010; Nakao et al. 2011; Rubia et al. 2013a). The very few published functional MRI (fMRI) comparisons between the disorders found taskdependent shared and disorder-specific deficits: shared dorsolateral prefrontal deficits during working memory (Chantiluke et al. in press a); shared dorsolateral prefronto-striato-parietal underactivation and reduced deactivation of posterior cingulate/precuneus default mode regions, but ASD-specific cerebellar overactivation during sustained attention (Christakou et al. 2013); ASD-specific underactivation in the ventromedial prefrontal cortex during reversal learning (Chantiluke et al. 2014a) and ADHD-specific orbitofrontal-striatal underactivation and ASD-specific left frontal overactivation during motor inhibition (Chantiluke et al. in press $b$ ). During temporal discounting, comorbid ADHD and ASD patients had unique brain-behaviour correlation abnormalities relative to controls in ventromedial and lateral frontolimbic regions, followed by the ASD group who had disorder-specific brain-behaviour correlation abnormalities in inferior frontotemporal regions (Chantiluke et al. 2014b). Finally, a recent resting-state fMRI study reported shared network centrality abnormalities in the precuneus, ADHD-specific increases in network centrality in the right striatum/pallidum and ASD-specific increases in network centrality in predominantly left temporolimbic areas (Di Martino et al. 2013).

Given the importance of establishing disorderspecific biomarkers in these two related disorders and evidence of the impact of long-term neurotropic medication on brain structure (Shaw et al. 2009; Murphy, 2010; Nakao et al. 2011; Rubia et al. 2013a), we investigated shared and disorder-specific GM and white matter (WM) abnormalities in 44 predominantly medication-naïve ADHD boys, 19 medication-naive ASD boys and 33 healthy boys.

\section{Method \\ Participants}

Forty-four mostly medication-naïve right-handed male adolescents with a clinical diagnosis of inattentive/ hyperactive-impulsive combined type ADHD, but not ASD, were recruited from out-patient clinics at the South London and Maudsley National Health Service (NHS) Foundation Trust. Diagnosis was assessed by a child psychiatrist using the standardized Maudsley Diagnostic Interview (MDI; Goldberg \& Murray, 2002), which assesses ADHD according to DSMIV-TR criteria (APA, 2000). All patients scored above the clinical cut-off for hyperactive-impulsive/inattentive symptoms on the parental Strengths and Difficulties Questionnaire (SDQ; Goodman, 1997) and the Conners' Parent Rating Scale (CPRS; Conners et al. 1998). ADHD patients were excluded if they scored above the clinical cut-off on the Social 
Communication Questionnaire (SCQ; Rutter et al. 2003). Most ADHD patients $(81.8 \%)$ were medication naïve, except for six patients $(13.6 \%)$ who received methylphenidate but had a wash-out of $48 \mathrm{~h}$ before scanning and two patients who had been treated with methylphenidate in the past. Nineteen righthanded medication-naïve male adolescents with a diagnosis of ASD, but not ADHD, were recruited through out-patient clinics. The ASD diagnosis was made using ICD-10 research diagnostic criteria (WHO, 1994), confirmed by the Autism Diagnostic Interview - Revised (ADI-R; Lord et al. 1994) and the Autism Diagnostic Observation Schedule (ADOS; Lord et al. 1989). ASD patients were excluded if they scored above 7 on the Hyperactivity/Inattention ratings on the SDQ. Five boys had high functioning autism (HFA) and 14 boys had Asperger's disorder. ADOS modules were selected based on verbal ability and age (Lord et al. 2000). Because of the high (verbal) functioning of the ASD boys, ADOS module 4 was used. Thirteen ASD boys reached the SCQ score cut-off. However, the algorithm used to obtain this score is not entirely indicative of clinical impairment and algorithms incorporating restricted and repetitive behaviours are more sensitive, as evidenced by the use of an algorithm that includes stereotyped behaviours in the new ADOS-2 (Lord et al. 2012). The ADI-R and ADOS are used in conjunction to obtain a holistic view and reliable diagnosis of an individual (Papanikolaou et al. 2009). All ASD participants scored above the clinical cut-off on the social, communication and restrictive and repetitive behaviour domains of the ADI-R and this, alongside the ADOS scores, was used to ensure that each adolescent met the criteria for ASD.

Thirty-three, age-matched, right-handed healthy boys were recruited through advertisement and scored below clinical thresholds on the SDQ and SCQ (Table 1). Participants were excluded if they had comorbid psychiatric disorders as assessed by the MDI, including learning disabilities, reading, speech or language disorder, neurological abnormalities, epilepsy, substance abuse and an $\mathrm{IQ}<70$ on the Wechsler Abbreviated Scale of Intelligence (WASI; Wechsler, 1999). Participants were reimbursed $£ 50$ for taking part in the study and written informed consent was obtained. The study was approved by the Camberwell St Giles Research Ethics Committee.

\section{MRI image acquisition}

Images were acquired using a 3-T GE Signa HDx system (General Electric, USA) at the Centre for Neuroimaging Sciences, Institute of Psychiatry, King's College London, UK. The body coil was used for radio frequency $(\mathrm{RF})$ transmission and an eight-channel head coil for RF reception. High-resolution structural three-dimensional (3D) T1-weighted magnetizationprepared rapid gradient-echo (MPRAGE) images were acquired. Full brain and skull coverage was required for each subject and detailed quality control was carried out on all MR images according to previously published quality control criteria (Simmons et al. 2011).

\section{VBM-DARTEL image preprocessing}

The images were first visually inspected for artefacts and structural abnormalities. Next, a VBM analysis (Ashburner \& Friston, 2000) was conducted to investigate group differences in GM volumes using SPM8 software (Statistical Parametric Mapping, Wellcome Department of Imaging Neuroscience, London, UK). The T1-weighted volumetric images were preprocessed using the VBM protocol with modulation (Ashburner, 2007), where the images were first segmented into GM, WM and cerebrospinal fluid (CSF). The DARTEL algorithm was applied to the segmented brain tissues to generate a study-specific template and to achieve an accurate inter-subject registration with improved realignment of smaller inner structures (Yassa \& Stark, 2009). The normalized modulated segmented GM/WM images were next affine transformed into Montreal Neurological Institute (MNI) space and smoothed with an isotropic Gaussian kernel of $8 \mathrm{~mm}$ at full-width half-maximum, providing a balance between predicted subcortical and cortical effects, and to accommodate the assumptions of Gaussian random field theory and the matched filter theorem.

\section{VBM analysis}

Group differences were evaluated for GM/WM volumes obtained in the tissue segmentation step of the VBMDARTEL preprocessing. The total brain volume (TBV) was calculated as the sum of GM and WM volumes. The normalized modulated and smoothed GM/WM images in each group were entered into voxel-wise ANOVAs using SPM8. We used a cluster-defining voxelwise threshold of $p<0.01$ (uncorrected) and a stringent cluster threshold of $p<0.05$ family-wise error (FWE) rate corrected for all the analyses. Cluster sizes were adjusted for smoothness non-uniformity by means of the VBM5.1 toolbox (Hayasaka et al. 2004). To test for correlations between structural abnormalities in GM and clinical symptoms, simple regression analyses were performed within each group.

\section{Results}

\section{Participant characteristics}

Groups did not differ significantly in age but there were significant differences in IQ $\left(F_{2,93}=27.3, p<\right.$ 
Table 1. Sample characteristics of participants

\begin{tabular}{lcccccc}
\hline Variables & CON (33) & ADHD (44) & ASD (19) & $F$ test & $p$ value $^{\mathrm{a}}$ & Subject contrast \\
\hline Age (years) & $14.3(2.52)$ & $13.6(1.87)$ & $14.9(1.86)$ & 2.68 & N.S. & - \\
IQ & $110(11.5)$ & $92.2(11.7)$ & $113(15.7)$ & 27.3 & $<0.001$ & ADHD $<$ ASD, CON \\
SDQ hyperactive/inattentive & $1.97(1.77)$ & $8.49(1.94)$ & $4.74(1.91)$ & 107.4 & $<0.001$ & ADHD $>$ ASD $>$ CON \\
SDQ emotional distress & $1.09(1.53)$ & $3.72(2.82)$ & $4.26(2.88)$ & 13.8 & $<0.001$ & ASD, ADHD>CON \\
SDQ conduct subscale & $0.72(1.49)$ & $5.67(2.42)$ & $2.16(1.95)$ & 55.0 & $<0.001$ & ADHD $>$ ASD $>$ CON \\
SDQ peer relationships & $0.88(1.31)$ & $3.77(2.37)$ & $6.21(2.30)$ & 43.0 & $<0.001$ & ASD $>$ ADHD $>$ CON \\
SDQ prosocial behaviour & $8.56(2.17)$ & $5.97(2.12)$ & $5.16(2.22)$ & 19.0 & $<0.001$ & CON $>$ ASD, ADHD \\
SDQ total scores & $4.66(4.53)$ & $21.6(5.88)$ & $17.4(5.46)$ & 91.7 & $<0.001$ & ADHD $>$ ASD $>$ CON \\
SCQ total score & $1.96(2.37)$ & $8.72(4.10)$ & $23.3(5.39)$ & 155.8 & $<0.001$ & ASD $>$ ADHD $>$ CON \\
CPRS-R total T score & $44.8(5.88)$ & $76.1(7.54)$ & $58.5(6.50)$ & 151.4 & $<0.001$ & ADHD $>$ ASD $>C O N$ \\
ADOS social scores & - & - & $7.35(3.92)$ & & & \\
ADOS communication scores & - & - & $2.24(1.48)$ & & & \\
ADOS communication and social scores & - & - & $9.59(5.03)$ & & & \\
ADOS stereotyped behaviour scores & - & - & $1.12(0.99)$ & & & \\
ADI-R social scores & - & - & $16.3(4.59)$ & & & \\
ADI-R communication scores & - & - & $13.9(3.77)$ & & &
\end{tabular}

IQ, Intelligence quotient as assessed with the Wechsler Abbreviated Scale of Intelligence (WASI); ADHD, attention deficit hyperactivity disorder; ASD, autism spectrum disorder; CON, controls; CPRS-R, revised Conners' Parent Rating Scale; SDQ, Strengths and Difficulties Questionnaire; SCQ, Social Communication Questionnaire; ADI-R, Autism Diagnostic Interview Revised; ADOS, Autism Diagnostic Observation Schedule; N.S., not significant.

Values given as mean (standard deviation).

${ }^{\text {a }}$ Bonferroni correction.

Table 2. Group differences between adolescents with ADHD, ASD and healthy controls in global brain volume

\begin{tabular}{lcccccc}
\hline & CON $(n=33)$ & ADHD $(n=44)$ & ASD $(n=19)$ & $F$ test & $p$ value $^{\mathrm{a}}$ & Subject contrast \\
\hline GM volume (ml) & $783(54.3)$ & $747(57.6)$ & $792(55.0)$ & 5.92 & 0.004 & ASD, CON $>$ ADHD \\
WM volume (ml) & $512(43.1)$ & $494(45.0)$ & $516(33.5)$ & 2.43 & 0.094 & - \\
TBV (ml) & $1295(95.8)$ & $1242(100.0)$ & $1307(86.6)$ & 4.41 & 0.015 & ASD, CON>ADHD \\
\hline
\end{tabular}

ADHD, Attention deficit hyperactivity disorder; ASD, autism spectrum disorder; CON, controls; GM, grey matter; WM, white matter; TBV, total brain volume (= GM volume + WM volumes).

Values given as mean (standard deviation).

${ }^{a}$ Bonferroni correction.

0.001) (Table 1). Post-hoc analyses showed that this was because the ADHD boys had lower IQs than healthy and ASD boys $(p<0.01)$, which is typical of the ADHD population (Kuntsi et al. 2004). When a covariate differs between groups because it is associated with a particular condition, and groups have not been selected randomly, an ANCOVA covarying for IQ to adjust for this variable would be inappropriate, as it would violate the basic ANCOVA assumption that the covariate is independent of the selected groups (Miller \& Chapman, 2001; Dennis et al. 2009). However, in our study, to assess the potential effect of IQ on GM group differences, GM volumes were correlated with
IQ within each group. As expected, based on the selection criteria, group differences were significant in CPRS, SDQ hyperactivity and SCQ scores (Table 1).

\section{VBM-DARTEL analysis of GM/WM volume differences}

There was a significant group difference in total GM volume and TBV (Table 2). Post-hoc analyses showed that ADHD boys had significantly smaller total GM volume and TBV compared to the other two groups. Hence, TBV was entered as a covariate in the subsequent analyses. 
Table 3. Group differences in GM volumes between adolescents with ADHD, ASD and healthy controls

\begin{tabular}{|c|c|c|c|c|c|}
\hline Brain region & BA & $\begin{array}{l}\text { Peak MNI } \\
\text { coordinates } \\
(\mathrm{x}, \mathrm{y}, \mathrm{z})\end{array}$ & $\begin{array}{l}\text { Number } \\
\text { of voxels }\end{array}$ & $\begin{array}{l}p \text { value of } \\
\text { cluster } \\
\text { (FWE corrected) }\end{array}$ & Subject contrast \\
\hline Right posterior cerebellum & - & $39,-67,-20$ & 2761 & 0.01 & $\mathrm{ASD}, \mathrm{CON}>\mathrm{ADHD}$ \\
\hline Left middle/superior temporal gyrus & $21 / 38$ & $-50,3,-17$ & 1956 & 0.04 & $\mathrm{ASD}>\mathrm{CON}, \mathrm{ADHD}^{\mathrm{a}}$ \\
\hline Left medial frontal gyrus & 6 & $-12,-9,57$ & 516 & $0.049^{*}$ & $\mathrm{ASD}>\mathrm{CON}^{\mathrm{a}}, \mathrm{ADHD}^{\mathrm{a}}$ \\
\hline
\end{tabular}

GM, Grey matter; ADHD, attention deficit hyperactivity disorder; ASD, autism spectrum disorder; BA, Brodmann area; MNI, Montreal Neurological Institute; CON, controls; FWE, family-wise error.

${ }^{a}$ At a lenient significance threshold of cluster $p<0.05$ uncorrected.

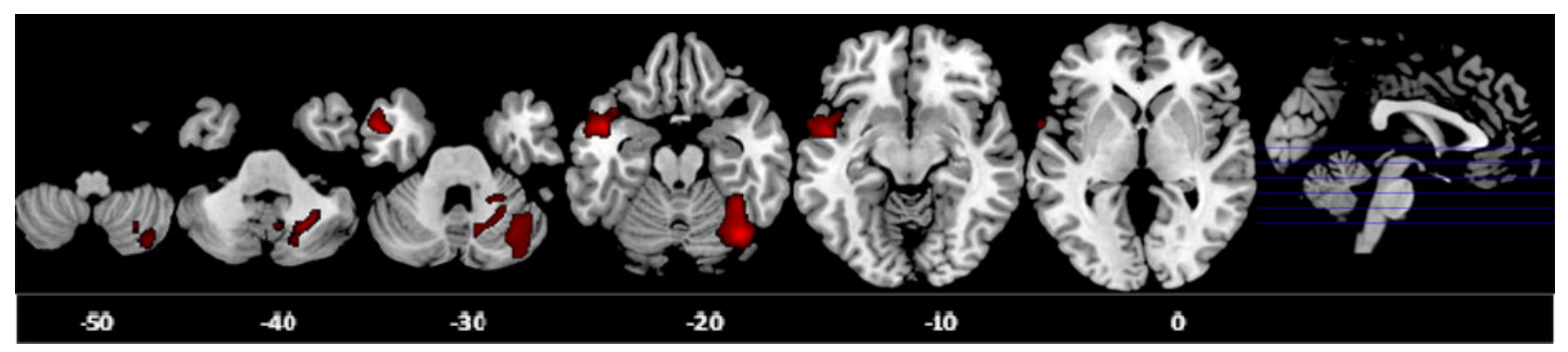

Fig. 1. Axial sections of grey matter (GM) reduction in the right posterior cerebellum in attention deficit hyperactivity disorder (ADHD) patients compared with controls and autism spectrum disorder (ASD) patients; and GM enlargement in the left middle/superior temporal gyrus in ASD patients relative to controls as revealed by the $F$ test $(p<0.05)$, family-wise error (FWE) corrected at the cluster level. Axial slices are marked with the $z$ coordinate as distance in millimetres from the anterior-posterior commissure. The right side of the image corresponds to the right side of the brain.

Voxel-wise ANCOVA $(p<0.05$ FWE-corrected $)$ showed a significant group effect in the GM volumes of the right posterior cerebellum [effect size $(E F)=$ 0.40, $\left.R^{2}=0.29\right]$ and left middle/superior temporal gyrus (MTG/STG: EF=0.17, $R^{2}=0.14$ ). Post-hoc analyses showed that ADHD boys had significantly smaller right posterior cerebellar GM compared to the other two groups, which did not differ from each other. ASD boys had significantly larger GM in the left MTG/STG compared with controls. At a more lenient cluster threshold of $p<0.05$ uncorrected, this was also significant relative to ADHD boys; in addition, ASD boys had a larger left medial frontal GM volume compared to both groups (Table 3, Fig. 1).

Given that one of the most significant sMRI findings in ADHD is that of reduced GM volumes in the basal ganglia (Nakao et al. 2011; Frodl \& Skokauskas, 2012), we conducted an additional ROI analysis extracting data for the bilateral basal ganglia using MARSBAR (Brett et al. 2002); no significant group differences were observed. The simple regression analyses between GM volumes and IQ and clinical ratings within each group revealed no significant correlation. No significant group differences were observed in WM volume.

Given that long-term stimulant medication is associated with more normal brain structure (Shaw et al.
2009; Murphy, 2010; Nakao et al. 2011; Rubia et al. 2013a), including the cerebellum (Bledsoe et al. 2009), we tested whether the cerebellum differences survived when we only compared the 36 medication-naive ADHD boys with the other two groups. The findings remained unchanged.

\section{Discussion}

To our knowledge, this is the second sMRI study comparing non-comorbid ADHD boys and non-comorbid ASD boys, in a relatively larger sample than the previous study (Brieber et al. 2007). Moreover, we included mostly medication-naïve participants and used a more stringent significance threshold corrected for multiple comparisons. The key finding is that noncomorbid, mostly medication-naïve, ADHD boys had a disorder-specific reduction in the right posterior cerebellar GM relative to non-comorbid medication-naïve ASD boys, suggesting that this may be a disorderspecific biomarker to differentiate between these two neurodevelopmental disorders. Furthermore, the finding survived when we only included the 36 medication-naïve ADHD boys in the analysis, thus excluding potential confounds of long-term stimulant medication treatment. ASD boys, by contrast, showed 
a GM enlargement in the left MTG/STG relative to controls, which was disorder specific relative to ADHD at a more lenient threshold.

The finding of an ADHD-specific GM deficit in the right posterior cerebellum extends prior literature on consistent deficits in ADHD in this region by showing for the first time that this is disorder specific relative to ASD. Reduction in cerebellar hemispheric volumes is one of the most consistent findings of sMRI studies in ADHD (Durston et al. 2004; Biederman et al. 2008; Montes et al. 2011; de Zeeuw et al. 2012; Lim et al. 2013), with the largest effect size in a meta-analysis of ROI sMRI studies (Valera et al. 2007). Reduced cerebellar volumes are also observed in longitudinal studies, where the deficit is sustained throughout adolescence (Castellanos et al. 2002; Mackie et al. 2007) and adulthood (Proal et al. 2011).

The cerebellum is one of the few brain regions that have been associated directly with ADHD diagnostic status and clinical outcome. Thus, ADHD patients with worse clinical outcome showed a progressively smaller total cerebellar volume with age, attributable mainly to the deviant trajectory of the inferiorposterior hemispheres, relative to healthy controls (Mackie et al. 2007). Furthermore, reduced (right) cerebellar volumes have been shown to be specifically associated with diagnostic status, rather than to be an endophenotype of ADHD, as deficits were not observed in unaffected siblings (Durston et al. 2004).

The cerebellum is one of the latest brain structures to fully develop. In particular, the cerebellar hemispheres reach their peak volume as late as around age 18 years, and the structural development of the different cerebellar regions parallels those prefrontal regions they are connected with to form the late-developing frontocerebellar networks that mediate higher-level motor, cognitive and affective functions (Mackie et al. 2007; Tiemeier et al. 2010; Arnsten \& Rubia, 2012). It is therefore plausible that our finding of a disorder-specific reduction in the right lateral cerebellar GM in ADHD boys relative to ASD boys, together with prior consistent evidence of smaller cerebellar hemisphere volumes in ADHD boys (Castellanos et al. 2002; Durston et al. 2004; Mackie et al. 2007; Valera et al. 2007; Biederman et al. 2008; Montes et al. 2011; Proal et al. 2011; de Zeeuw et al. 2012; Lim et al. 2013) could potentially reflect a maturational delay in ADHD. This would parallel the delay in GM thickness development of prefrontal and temporoparietal regions that co-develop with the cerebellum (Shaw et al. 2007, 2012).

The cerebellum has traditionally been considered to be primarily involved in motor control. However, lesion and fMRI studies have consistently demonstrated its involvement in a wide range of cognitive and affective functions, in particular sustained and shifting attention (Schmahmann, 2004), working memory (Ravizza et al. 2006), inhibitory control (Rubia et al. 2007, 2013b), temporal information processing (Rubia \& Smith, 2004; Rubia, 2006; Wiener et al. 2010; Noreika et al. 2012) and emotion regulation (Allen et al. 1997). This is further underscored by the extensive connections of the lateral cerebellar hemispheres to the prefrontal cortex and the basal ganglia, forming fronto-striato-cerebellar networks (Arnsten \& Rubia, 2012). Based on lesion studies (Exner et al. 2004) and meta-analyses (Stoodley \& Schmahmann, 2009), the anterior part of the cerebellum is particularly involved in motor and sensory functions, the medial part in emotion processes and the lateral posterior region, found to be abnormal in ADHD in this study, in higher-level cognitive abilities such as attention (Kellermann et al. 2012; Li et al. 2012), inhibition (Rubia et al. 2007, 2013b), working memory (Stoodley \& Schmahmann, 2010; Massat et al. 2012; Stoodley et al. 2012) and timing functions (O'Reilly et al. 2008; Wiener et al. 2010).

The right-hemispheric location of the cerebellar GM deficit finding in ADHD is also important, given that the right posterior cerebellar hemisphere has been found to be particularly relevant for attention and working memory (Kellermann et al. 2012; Li et al. 2012; Bernard \& Seidler, 2013). ADHD children have consistent deficits in these above-mentioned cognitive functions, especially working memory, sustained attention (Willcutt et al. 2005; Rubia, 2011; Cubillo et al. 2012a), inhibition (Lijffijt et al. 2005) and timing functions (Rubia et al. 2009a; Noreika et al. 2012). In ASD, impairments in these functions are more controversial, with many negative findings with respect to selective and sustained attention (Johnson et al. 2007; Rommelse et al. 2011) and working memory (Rommelse et al. 2011), and less consistent evidence for inhibition (Rommelse et al. 2011) and timing impairment (Falter et al. 2012). Furthermore, when ADHD comorbidity is excluded and compared to ADHD, ASD patients are less impaired in these cognitive functions (Johnson et al. 2007; Rommelse et al. 2011).

Evidence for cerebellar GM abnormalities in ADHD are further supported by diffusion tensor imaging (DTI) studies that have reported reduced fractional anisotropy (FA) in the WM tracts of the right middle (Bechtel et al. 2009; Kobel et al. 2010; Chuang et al. 2013) and left inferior cerebellar peduncle (Nagel et al. 2011) in ADHD patients compared to controls, suggesting deficient structural connectivity between the cerebellum and prefrontal regions.

Cerebellar GM deficits in ADHD also echo evidence for abnormal function of this region based on fMRI studies that have found the lateral and medial cerebellum to be abnormal in their activation in ADHD patients 
together with frontostriatal deficits, most consistently during tasks of sustained and selective attention (Rubia et al. 2009b; Cubillo et al. 2012a), timing (Rubia et al. 2009a; Valera et al. 2010; Vloet et al. 2010; Hart et al. 2012) and inhibition (Rubia et al. 2011, 2013a; Cubillo et al. 2012b; Hart et al. 2014).

Similarly, fMRI studies have detected abnormal functional connectivity between the cerebellum and prefrontal, striatal and parietal regions in ADHD patients during attention, timing (Rubia et al. 2009b; Vloet et al. 2010) and working memory (Massat et al. 2012) performance, suggesting that different task-relevant fronto-striatocerebellar networks are dysfunctional in ADHD.

The disorder specificity of the right posterior cerebellar GM deficit relative to ASD is intriguing. Although cerebellar abnormalities are consistent findings in sMRI studies of ASD, there is debate regarding the nature and consistency of these cerebellar alterations. Some studies have found the cerebellum to be enlarged (Palmen et al. 2005; Bonilha et al. 2008), smaller (Webb et al. 2009; Toal et al. 2010) or not to differ compared with controls (Hazlett et al. 2005; Scott et al. 2009). The age of ASD patients is likely to play an important role given that, in early infancy and childhood, ASD is associated with significantly enlarged GM volumes relative to controls; later on, in adolescence and adulthood, there is evidence for arrested growth relative to controls (Amaral et al. 2008). By adolescence, some studies found normal TBV (Hazlett et al. 2005; Scott et al. 2009), suggesting that the precocious overgrowth from the first years of life may normalize with age by adolescence. However, most of this evidence is based on cross-sectional data, and longitudinal data are needed to elucidate developmental growth trajectories. Our findings, however, suggest that, by adolescence, the cerebellar hemispheres are disorder-specifically reduced in ADHD relative to ASD who have normal TBV and cerebellar GM at this age point.

Importantly, we found that the ADHD-specific GM deficit relative to the other two groups in the right posterior cerebellum survived when we only included medication-naïve patients. Medication naivety is crucial for neuroimaging studies, as we have shown in a meta-regression analysis that long-term stimulant medication is associated with more normal brain structure in the basal ganglia in ADHD (Nakao et al. 2011). Retrospective analyses have found that medicationnaïve ADHD patients have more abnormal GM than long-term medicated ADHD patients in the cerebellum (Bledsoe et al. 2009) and other ADHD-relevant areas (Shaw et al. 2009; Ivanov et al. 2010; Rubia et al. 2013a). Therefore, long-term stimulant medication is not a confound in our results.

ASD boys also had larger left MTG/STG GM volumes relative to controls, and at a more lenient threshold relative to ADHD boys. The STG is involved in auditory processing including language and has been implicated in social cognition (Pelphrey et al. 2004). Language deficits are a core feature of ASD and failure to develop normal language comprehension is an early warning sign of autism (Eyler et al. 2012). Several studies have reported larger left temporal GM volume in ASD patients relative to healthy controls (Hazlett et al. 2006; Rojas et al. 2006; Knaus et al. 2009; Verhoeven et al. 2010; Cauda et al. 2011), which was also correlated with social and communication deficits (Rojas et al. 2006; Verhoeven et al. 2010). In addition, one study has shown that ASD patients, compared to healthy controls, did not show the normal age-related reductions in MTG/STG cortical volume and thickness during adolescence and adulthood, suggesting cortical dysmaturation in a brain region that is crucial to social cognition and language (Raznahan et al. 2010). Several fMRI studies have suggested that abnormal activation in the left MTG/STG [especially Brodmann area (BA) 21, as found in this study] may play a central role in the typical language impairment in ASD (Redcay \& Courchesne, 2008; Eyler et al. 2012). In particular, a failure of the left temporal cortex to specialize for language during early development may reflect a fundamental early neural developmental pathology in autism (Eyler et al. 2012). Furthermore, a DTI study reported abnormalities in the microstructural organization of the WM tracts involving the STG and temporal stem in autistic patients compared to controls (Lee et al. 2007). Our finding of a larger STG GM volume in ASD parallels the findings of Brieber et al. (2007), who reported an increased GM volume in the adjacent right supramarginal gyrus in ASD relative to controls and ADHD, although their findings did not survive correction for multiple comparisons. In addition, our finding of abnormalities in the left temporal gyrus in ASD patients parallels the findings of Di Martino et al. (2013), who found disorder-specific functional network abnormalities in ASD relative to ADHD and controls in limbic networks, including the left planum temporale and temporal cortex. The disorder specificity of this abnormality may only have been detected at a more lenient threshold because of the relatively smaller numbers in the ASD group; replications in larger samples are therefore needed to corroborate these findings. The relatively small numbers of participants may also have prevented us from finding a structural deficit in the basal ganglia in ADHD, which was observed in two meta-analyses of sMRI studies (Nakao et al. 2011; Frodl \& Skokauskas, 2012).

We found no WM differences between groups. sMRI findings have been inconclusive, with some studies finding WM abnormalities in ADHD (Castellanos 
et al. 2002; Durston et al. 2004) and ASD (McAlonan et al. 2005; Bonilha et al. 2008), but not others (Palmen et al. 2005; Brun et al. 2009; Batty et al. 2010). The sample sizes, especially for the ASD group, may have been too small to detect any WM abnormalities. Moreover, changes in WM integrity may be assessed more accurately using DTI (Whitwell, 2009).

A strength of this study is that all ASD and most ADHD patients were medication naïve. Furthermore, our findings survived a subanalysis in only medication-naïve ADHD patients. This is important because stimulant medication, the gold standard medication for ADHD, and selective serotonin reuptake inhibitors (SSRIs), which are sometimes used in ASD, have been associated with differences in brain structure including the cerebellum (Bledsoe et al. 2009; Ivanov et al. 2010; Murphy, 2010; Nakao et al. 2011; Rubia et al. 2013a). Another strength is the careful diagnosis of ASD patients who were non-comorbid with ADHD, using the ICD-10, ADI and ADOS, and of ADHD patients who were non-comorbid with ASD, using the CPRS, SDQ and SCQ, both without other comorbid psychiatric diagnoses. Although the inclusion of only males enhances the homogeneity of the group and is based on the higher prevalence in boys for both disorders (Rommelse et al. 2010), it limits the generalizability to females with the disorders. Furthermore, we included only high-functioning adolescents with ASD and Asperger's disorder, and the combined subtype of ADHD, which limits the generalizability to other subtypes within ASD or ADHD. Additionally, although in this study we carefully excluded comorbidity between the two disorders, future studies should elucidate to what extent the comorbid conditions share the same deficits observed in non-comorbid ADHD and non-comorbid ASD or whether they are different in their neurobiological substrates. Another limitation is the relatively smaller sample size of the ASD group, although it was still somewhat larger than that of the only other study that compared brain structure between these two disorders (Brieber et al. 2007). Finally, in view of evidence for increased testosterone levels in ASD during puberty (Geier \& Geier, 2007), future studies should include measures of pubertal status and hormonal measures.

In summary, using a stringent threshold corrected for multiple comparisons and including mostly medication-naïve, carefully diagnosed non-comorbid groups of ADHD and ASD boys, we found that ADHD boys had a disorder-specific GM volume reduction in the right posterior cerebellum whereas ASD boys had a disorder-specific GM volume enlargement in the left MTG/STG, albeit at a more lenient significance level. The findings represent a first step towards the delineation of disorder-specific structural biomarkers for these two related disorders.

\section{Acknowledgements}

Data collection was supported by grants from the National Institute for Health Research (NIHR) Biomedical Research Centre (BRC) for Mental Health at South London and Maudsley NHS Foundation Trust (SLaM), the Institute of Psychiatry, King's College London, and Lilly Pharmaceuticals. L.L. was supported by the National Medical Research Council (Singapore); Kids Company London and the Rita Lila Weston Foundation. K.C., A.I.C., A.B.S. and A.S. and were supported by the NIHR BRC.

\section{Declaration of Interest}

K.R. has received speakers' honoraria from Lilly and Shire. The other authors have no conflicts of interest to declare.

\section{References}

Allen G, Buxton RB, Wong EC, Courchesne E (1997). Attentional activation of the cerebellum independent of motor involvement. Science 275, 1940-1943.

Amaral DG, Schumann CM, Nordahl CW (2008). Neuroanatomy of autism. Trends in Neurosciences 31, 137-145. APA (2000). Diagnostic and Statistical Manual of Mental Disorders, Fourth Edition, Text Revision. DSM-IV-TR. American Psychiatric Association: Washington, DC. APA (2013). Diagnostic and Statistical Manual of Mental Disorders, Fifth Edition, DSM-5. American Psychiatric Association: Arlington, VA.

Arnsten AF, Rubia K (2012). Neurobiological circuits regulating attention, cognitive control, motivation, and emotion: disruptions in neurodevelopmental psychiatric disorders. Journal of the American Academy of Child and Adolescent Psychiatry 51, 356-367.

Ashburner J (2007). A fast diffeomorphic image registration algorithm. Neurolmage 38, 95-113.

Ashburner J, Friston KJ (2000). Voxel-based morphometry the methods. NeuroImage 11, 805-821.

Batty MJ, Liddle EB, Pitiot A, Toro R, Groom MJ, Scerif G, Liotti M, Liddle PF, Paus T, Hollis C (2010). Cortical gray matter in attention-deficit/hyperactivity disorder: a structural magnetic resonance imaging study. Journal of the American Academy of Child and Adolescent Psychiatry 49, 229-238.

Bechtel N, Kobel M, Penner IK, Klarhofer M, Scheffler K, Opwis K, Weber P (2009). Decreased fractional anisotropy in the middle cerebellar peduncle in children with epilepsy and/or attention deficit/hyperactivity disorder: a preliminary study. Epilepsy and Behavior 15, 294-298.

Bernard JA, Seidler RD (2013). Cerebellar contributions to visuomotor adaptation and motor sequence learning: an ALE meta-analysis. Frontiers in Human Neuroscience 7, 27. 
Biederman J, Makris N, Valera EM, Monuteaux MC, Goldstein JM, Buka S, Boriel DL, Bandyopadhyay S, Kennedy DN, Caviness VS, Bush G, Aleardi M, Hammerness P, Faraone SV, Seidman LJ (2008). Towards further understanding of the comorbidity between attention deficit hyperactivity disorder and bipolar disorder: a MRI study of brain volumes. Psychological Medicine 38, 1045-1056.

Bledsoe J, Semrud-Clikeman M, Pliszka SR (2009). A magnetic resonance imaging study of the cerebellar vermis in chronically treated and treatment-naive children with attention-deficit/hyperactivity disorder combined type. Biological Psychiatry 65, 620-624.

Bonilha L, Cendes F, Rorden C, Eckert M, Dalgalarrondo P, Li M, Steiner CE (2008). Gray and white matter imbalance - typical structural abnormality underlying classic autism? Brain and Development 30, 396-401.

Brett M, Johnsrude IS, Owen AM (2002). The problem of functional localization in the human brain. Nature Reviews Neuroscience 3, 243-249.

Brieber S, Neufang S, Bruning N, Kamp-Becker I, Remschmidt H, Herpertz-Dahlmann B, Fink GR, Konrad K (2007). Structural brain abnormalities in adolescents with autism spectrum disorder and patients with attention deficit/ hyperactivity disorder. Journal of Child Psychology and Psychiatry and Allied Disciplines 48, 1251-1258.

Brun CC, Nicolson R, Lepore N, Chou YY, Vidal CN, DeVito TJ, Drost DJ, Williamson PC, Rajakumar N, Toga AW, Thompson PM (2009). Mapping brain abnormalities in boys with autism. Human Brain Mapping 30, 3887-3900.

Castellanos FX, Lee PP, Sharp W, Jeffries NO, Greenstein DK, Clasen LS, Blumenthal JD, James RS, Ebens CL, Walter JM, Zijdenbos A, Evans AC, Giedd JN, Rapoport JL (2002). Developmental trajectories of brain volume abnormalities in children and adolescents with attention-deficit/hyperactivity disorder. Journal of the American Medical Association 288, 1740-1748.

Cauda F, Geda E, Sacco K, D'Agata F, Duca S, Geminiani G, Keller R (2011). Grey matter abnormality in autism spectrum disorder: an activation likelihood estimation meta-analysis study. Journal of Neurology, Neurosurgery and Psychiatry 82, 1304-1313.

Chantiluke K, Barrett N, Giampietro V, Brammer M, Simmons A, Murphy DG, Rubia K (2014a). Inverse effect of fluoxetine on medial prefrontal cortex activation during reward reversal in ADHD and autism. Cerebral Cortex. Published online: 22 January 2014. doi:10.1093/cercor/ bht365.

Chantiluke K, Barrett N, Giampietro V, Brammer M, Simmons A, Murphy DG, Rubia K (in press a). Disorder-dissociated effects of fluoxetine on brain function of working memory in attention deficit hyperactivity disorder and autism spectrum disorder. Psychological Medicine.

Chantiluke K, Barrett N, Giampietro V, Santosh P, Brammer M, Simmons A, Murphy D, Rubia K (in press $b$ ). Inverse fluoxetine effects on inhibitory control in non-comorbid boys with ADHD and with ASD. Psychopharmacology.
Chantiluke K, Christakou A, Murphy CM, Giampietro V, Daly EM, Ecker C, Brammer M, Murphy DG, the MRC AIMS Consortium, Rubia K (2014b). Disorder-specific functional abnormalities during temporal discounting in youth with attention deficit hyperactivity disorder (ADHD), autism and comorbid ADHD and autism. Psychiatry Research 223, 113-120.

Christakou A, Murphy CM, Chantiluke K, Cubillo AI, Smith AB, Giampietro V, Daly E, Ecker C, Robertson D, Murphy DG, Rubia K (2013). Disorder-specific functional abnormalities during sustained attention in youth with attention deficit hyperactivity disorder (ADHD) and with autism. Molecular Psychiatry 18, 236-244.

Chuang TC, Wu MT, Huang SP, Weng MJ, Yang P (2013). Diffusion tensor imaging study of white matter fiber tracts in adolescent attention-deficit/hyperactivity disorder. Psychiatry Research 211, 186-187.

Conners CK, Sitarenios G, Parker JDA, Epstein JN (1998). Revision and restandardization of the Conners Teacher Rating Scale (CTRS-R): factor structure, reliability, and criterion validity. Journal of Abnormal Child Psychology 26, 279-291.

Courchesne E, Campbell K, Solso S (2011). Brain growth across the life span in autism: age-specific changes in anatomical pathology. Brain Research 1380, 138-145.

Cubillo A, Halari R, Smith A, Taylor E, Rubia K (2012a). A review of fronto-striatal and fronto-cortical brain abnormalities in children and adults with attention deficit hyperactivity disorder (ADHD) and new evidence for dysfunction in adults with ADHD during motivation and attention. Cortex 48, 194-215.

Cubillo A, Smith AB, Barrett N, Giampietro V, Brammer MJ, Simmons A, Rubia K (2012b). Shared and drug-specific effects of atomoxetine and methylphenidate on inhibitory brain dysfunction in medication-naive ADHD boys. Cerebral Cortex 24, 174-185.

de Zeeuw P, Zwart F, Schrama R, van Engeland H, Durston S (2012). Prenatal exposure to cigarette smoke or alcohol and cerebellum volume in attention-deficit/hyperactivity disorder and typical development. Translational Psychiatry 2, e84.

Dennis M, Francis DJ, Cirino PT, Schachar R, Barnes MA, Fletcher JM (2009). Why IQ is not a covariate in cognitive studies of neurodevelopmental disorders. Journal of the International Neuropsychological Society 15, 331-343.

Di Martino A, Zuo X-N, Kelly C, Grzadzinski R, Mennes M, Schvarcz A, Rodman J, Lord C, Castellanos FX, Milham MP (2013). Shared and distinct intrinsic functional network centrality in autism and attention-deficit/hyperactivity disorder. Biological Psychiatry 74, 623-632.

Durston S, Hulshoff Pol HE, Schnack HG, Buitelaar JK, Steenhuis MP, Minderaa RB, Kahn RS, van Engeland $\mathbf{H}$ (2004). Magnetic resonance imaging of boys with attention-deficit/hyperactivity disorder and their unaffected siblings. Journal of the American Academy of Child and Adolescent Psychiatry 43, 332-340.

Exner C, Weniger G, Irle E (2004). Cerebellar lesions in the PICA but not SCA territory impair cognition. Neurology 63 2132-2135. 
Eyler LT, Pierce K, Courchesne E (2012). A failure of left temporal cortex to specialize for language is an early emerging and fundamental property of autism. Brain 135, 949-960.

Falter CM, Noreika V, Wearden JH, Bailey AJ (2012). More consistent, yet less sensitive: interval timing in autism spectrum disorders. Quarterly Journal of Experimental Psychology 65, 2093-2107.

Frodl T, Skokauskas N (2012). Meta-analysis of structural MRI studies in children and adults with attention deficit hyperactivity disorder indicates treatment effects. Acta Psychiatrica Scandinavica 125, 114-126.

Geier DA, Geier MR (2007). A prospective assessment of androgen levels in patients with autistic spectrum disorders: biochemical underpinnings and suggested therapies. Neuroendocrinology Letters 28, 565-573.

Geurts HM, Verte S, Oosterlaan J, Roeyers H, Sergeant JA (2004). How specific are executive functioning deficits in attention deficit hyperactivity disorder and autism? Journal of Child Psychology and Psychiatry and Allied Disciplines 45, 836-854.

Goldberg D, Murray R (eds) (2002). The Maudsley Handbook of Practical Psychiatry. Oxford University Press: Oxford.

Goodman R (1997). The strengths and difficulties questionnaire: a research note. Journal of Child Psychology and Psychiatry and Allied Disciplines 38, 581-586.

Hart H, Chantiluke K, Cubillo AI, Smith AB, Simmons A, Brammer MJ, Marquand AF, Rubia K (2014). Pattern classification of response inhibition in ADHD: toward the development of neurobiological markers for ADHD. Human Brain Mapping 35, 3083-3094.

Hart H, Radua J, Mataix-Cols D, Rubia K (2012). Meta-analysis of fMRI studies of timing in attention-deficit hyperactivity disorder (ADHD). Neuroscience and Biobehavioral Reviews 36, 2248-2256.

Hayasaka S, Phan KL, Liberzon I, Worsley KJ, Nichols TE (2004). Nonstationary cluster-size inference with random field and permutation methods. NeuroImage 22, 676-687.

Hazlett HC, Poe M, Gerig G, Smith RG, Provenzale J, Ross A, Gilmore J, Piven J (2005). Magnetic resonance imaging and head circumference study of brain size in autism: birth through age 2 years. Archives of General Psychiatry 62, 1366-1376.

Hazlett HC, Poe MD, Gerig G, Smith RG, Piven J (2006). Cortical gray and white brain tissue volume in adolescents and adults with autism. Biological Psychiatry 59, 1-6.

Hyde KL, Samson F, Evans AC, Mottron L (2010). Neuroanatomical differences in brain areas implicated in perceptual and other core features of autism revealed by cortical thickness analysis and voxel-based morphometry. Human Brain Mapping 31, 556-566.

Ivanov I, Bansal R, Hao X, Zhu H, Kellendonk C, Miller L, Sanchez-Pena J, Miller AM, Chakravarty MM, Klahr K, Durkin K, Greenhill LL, Peterson BS (2010).

Morphological abnormalities of the thalamus in youths with attention deficit hyperactivity disorder. American Journal of Psychiatry 167, 397-408.

Johnson KA, Robertson IH, Kelly SP, Silk TJ, Barry E, Daibhis A, Watchorn A, Keavey M, Fitzgerald $M$, Gallagher L, Gill M, Bellgrove MA (2007). Dissociation in performance of children with ADHD and high-functioning autism on a task of sustained attention. Neuropsychologia $\mathbf{4 5}$, 2234-2245.

Kellermann T, Regenbogen C, De Vos M, Mossnang C, Finkelmeyer A, Habel U (2012). Effective connectivity of the human cerebellum during visual attention. Journal of Neuroscience 32, 11453-11460.

Knaus TA, Silver AM, Dominick KC, Schuring MD, Shaffer N, Lindgren KA, Joseph RM, Tager-Flusberg H (2009). Age-related changes in the anatomy of language regions in autism spectrum disorder. Brain Imaging and Behavior 3, 51-63.

Kobel M, Bechtel N, Specht K, Klarhofer M, Weber P, Scheffler K, Opwis K, Penner IK (2010). Structural and functional imaging approaches in attention deficit/ hyperactivity disorder: does the temporal lobe play a key role? Psychiatry Research 183, 230-236.

Kuntsi J, Eley TC, Taylor A, Hughes C, Asherson P, Caspi A, Moffitt TE (2004). Co-occurrence of ADHD and low IQ has genetic origins. American Journal of Medical Genetics. Part B, Neuropsychiatric Genetics 124B, 41-47.

Lee PS, Foss-Feig J, Henderson JG, Kenworthy LE, Gilotty L, Gaillard WD, Vaidya CJ (2007). Atypical neural substrates of Embedded Figures Task performance in children with autism spectrum disorder. NeuroImage 38, 184-193.

Li X, Sroubek A, Kelly MS, Lesser I, Sussman E, He Y, Branch C, Foxe JJ (2012). Atypical pulvinar-cortical pathways during sustained attention performance in children with attention-deficit/hyperactivity disorder. Journal of the American Academy of Child and Adolescent Psychiatry 51, 1197-1207.e4.

Lijffijt M, Kenemans JL, Verbaten MN, van Engeland H (2005). A meta-analytic review of stopping performance in attention-deficit/hyperactivity disorder: deficient inhibitory motor control? Journal of Abnormal Psychology 114, 216-222.

Lim L, Marquand A, Cubillo AA, Smith AB, Chantiluke K, Simmons A, Mehta M, Rubia K (2013). Disorder-specific predictive classification of adolescents with attention deficit hyperactivity disorder (ADHD) relative to autism using structural magnetic resonance imaging. PLOS ONE 8, e63660.

Lord C, Risi S, Lambrecht L, Cook EH Jr., Leventhal BL, DiLavore PC, Pickles A, Rutter M (2000). The Autism Diagnostic Observation Schedule-Generic: a standard measure of social and communication deficits associated with the spectrum of autism. Journal of Autism and Developmental Disorders 30, 205-223.

Lord C, Rutter M, DiLavore P, Risi S, Gotham K, Bishop SL (2012). Autism Diagnostic Observation Schedule. Western Psychological Services: Los Angeles, CA.

Lord C, Rutter M, Goode S, Heemsbergen J, Jordan H, Mawhood L, Schopler E (1989). Autism Diagnostic Observation Schedule: a standardized observation of communicative and social behavior. Journal of Autism and Developmental Disorders 19, 185-212.

Lord C, Rutter M, Le Couteur A (1994). Autism Diagnostic Interview-Revised: a revised version of a diagnostic interview for caregivers of individuals with possible 
pervasive developmental disorders. Journal of Autism and Developmental Disorders 24, 659-685.

Mackie S, Shaw P, Lenroot R, Pierson R, Greenstein DK, Nugent TF, Sharp WS, Giedd JN, Rapoport JL (2007). Cerebellar development and clinical outcome in attention deficit hyperactivity disorder. American Journal of Psychiatry 164, 647-655.

Massat I, Slama H, Kavec M, Linotte S, Mary A, Baleriaux D, Metens T, Mendlewicz J, Peigneux P (2012). Working memory-related functional brain patterns in never medicated children with ADHD. PLoS ONE 7, e49392.

McAlonan GM, Cheung V, Cheung C, Suckling J, Lam GY, Tai KS, Yip L, Murphy DG, Chua SE (2005). Mapping the brain in autism. A voxel-based MRI study of volumetric differences and intercorrelations in autism. Brain 128, 268-276.

Miller GA, Chapman JP (2001). Misunderstanding analysis of covariance. Journal of Abnormal Psychology 110, 40-48.

Montes LG, Ricardo-Garcell J, De la Torre LB, Alcantara HP, Garcia RB, Acosta DA, Bouzas AF (2011). Cerebellar gray matter density in females with ADHD combined type: a cross-sectional voxel-based morphometry study. Journal of Attention Disorders 15, 368-381.

Murphy SE (2010). Using functional neuroimaging to investigate the mechanisms of action of selective serotonin reuptake inhibitors (SSRIs). Current Pharmaceutical Design 16, 1990-1997.

Nagel BJ, Bathula D, Herting M, Schmitt C, Kroenke CD, Fair D, Nigg JT (2011). Altered white matter microstructure in children with attention-deficit/hyperactivity disorder. Journal of the American Academy of Child and Adolescent Psychiatry 50, 283-292.

Nakao T, Radua J, Rubia K, Mataix-Cols D (2011). Gray matter volume abnormalities in ADHD: voxel-based meta-analysis exploring the effects of age and stimulant medication. American Journal of Psychiatry 168, 1154-1163.

Nickl-Jockschat T, Habel U, Michel TM, Manning J, Laird AR, Fox PT, Schneider F, Eickhoff SB (2012). Brain structure anomalies in autism spectrum disorder - a meta-analysis of VBM studies using anatomic likelihood estimation. Human Brain Mapping 33, 1470-1489.

Noreika V, Falter CM, Rubia K (2012). Timing deficits in attention-deficit/hyperactivity disorder (ADHD): evidence from neurocognitive and neuroimaging studies. Neuropsychologia 51, 235-266.

O'Reilly JX, Mesulam MM, Nobre AC (2008). The cerebellum predicts the timing of perceptual events. Journal of Neuroscience 28, 2252-2260.

Palmen SJ, Hulshoff Pol HE, Kemner C, Schnack HG, Durston S, Lahuis BE, Kahn RS, Van Engeland H (2005). Increased gray-matter volume in medication-naive high-functioning children with autism spectrum disorder. Psychological Medicine 35, 561-570.

Papanikolaou K, Paliokosta E, Houliaras G, Vgenopoulou S, Giouroukou E, Pehlivanidis A, Tomaras V, Tsiantis I (2009). Using the Autism Diagnostic Interview-Revised and the Autism Diagnostic Observation Schedule-Generic for the diagnosis of autism spectrum disorders in a Greek sample with a wide range of intellectual abilities. Journal of Autism and Developmental Disorders 39, 414-420.
Pelphrey KA, Viola RJ, McCarthy G (2004). When strangers pass: processing of mutual and averted social gaze in the superior temporal sulcus. Psychological Science 15, 598-603.

Proal E, Reiss PT, Klein RG, Mannuzza S, Gotimer K, Ramos-Olazagasti MA, Lerch JP, He Y, Zijdenbos A, Kelly C, Milham MP, Castellanos FX (2011). Brain gray matter deficits at 33-year follow-up in adults with attention-deficit/hyperactivity disorder established in childhood. Archives of General Psychiatry 68, 1122-1134.

Ravizza SM, McCormick CA, Schlerf JE, Justus T, Ivry RB, Fiez JA (2006). Cerebellar damage produces selective deficits in verbal working memory. Brain 129, 306-320.

Raznahan A, Toro R, Daly E, Robertson D, Murphy C, Deeley Q, Bolton PF, Paus T, Murphy DG (2010). Cortical anatomy in autism spectrum disorder: an in vivo MRI study on the effect of age. Cerebral Cortex 20, 1332-1340.

Redcay E, Courchesne E (2008). Deviant functional magnetic resonance imaging patterns of brain activity to speech in 2-3-year-old children with autism spectrum disorder. Biological Psychiatry 64, 589-598.

Rojas DC, Peterson E, Winterrowd E, Reite ML, Rogers SJ, Tregellas JR (2006). Regional gray matter volumetric changes in autism associated with social and repetitive behavior symptoms. BMC Psychiatry 6, 56.

Rommelse NN, Franke B, Geurts HM, Hartman CA, Buitelaar JK (2010). Shared heritability of attention-deficit/ hyperactivity disorder and autism spectrum disorder. European Child and Adolescent Psychiatry 19, 281-295.

Rommelse NN, Geurts HM, Franke B, Buitelaar JK, Hartman CA (2011). A review on cognitive and brain endophenotypes that may be common in autism spectrum disorder and attention-deficit/hyperactivity disorder and facilitate the search for pleiotropic genes. Neuroscience and Biobehavioral Reviews 35, 1363-1396.

Rubia K (2006). The neural correlates of timing functions. In Timing the Future: The Case for a Time-based Prospective Memory (ed. J. Glicksohn and M. Myslobodsky), pp. 213238. World Scientific Publishing: Hackensack, NJ.

Rubia K (2011). 'Cool' inferior frontostriatal dysfunction in attention-deficit/hyperactivity disorder versus 'hot' ventromedial orbitofrontal-limbic dysfunction in conduct disorder: a review. Biological Psychiatry 69, e69-e87.

Rubia K, Alegria A, Brinson H (2014). Imaging the ADHD brain: disorder-specificity, medication effects and clinical translation. Expert Review of Neurotherapeutics 14, 519-538.

Rubia K, Alzamora A, Cubillo A, Smith A, Brammer M, Radua J (2013a). Effects of stimulants on brain function in ADHD: a systematic review and meta-analysis. Biological Psychiatry. Published online: 24 October 2013. doi:10.1016/j. biopsych.2013.10.016.

Rubia K, Halari R, Christakou A, Taylor E (2009a). Impulsiveness as a timing disturbance: neurocognitive abnormalities in attention-deficit hyperactivity disorder during temporal processes and normalization with methylphenidate. Philosophical Transactions of the Royal Society of London. Series B, Biological Sciences 364, 1919-1931.

Rubia K, Halari R, Cubillo A, Mohammad M, Taylor E (2009b). Methylphenidate normalises activation and functional connectivity deficits in attention and motivation 
networks in medication-naïve children with ADHD during a rewarded continuous performance task.

Neuropharmacology 57, 640-652.

Rubia K, Halari R, Mohammad AM, Taylor E, Brammer M (2011). Methylphenidate normalizes frontocingulate underactivation during error processing in attention-deficit/ hyperactivity disorder. Biological Psychiatry 70, 255-262.

Rubia K, Lim L, Ecker C, Halari R, Giampietro V, Simmons A, Brammer M, Smith A (2013b). Effects of age and gender on neural networks of motor response inhibition: from adolescence to mid-adulthood. Neurolmage 83, 690-703.

Rubia K, Smith A (2004). The neural correlates of cognitive time management: a review. Acta Neurobiologiae Experimentalis 64, 329-340.

Rubia K, Smith A, Taylor E, Brammer M (2007). Linear age-correlated functional development of right inferior fronto-striato-cerebellar networks during response inhibition and anterior cingulate during error-related processes. Human Brain Mapping 28, 1163-1177.

Rutter M, Bailey AJ, Lord C (2003). Social Communication Questionnaire. Western Psychological Services:

Los Angeles, CA.

Schatz AM, Weimer AK, Trauner DA (2002). Brief report: attention differences in Asperger syndrome. Journal of Autism and Developmental Disorders 32, 333-336.

Schmahmann JD (2004). Disorders of the cerebellum: ataxia, dysmetria of thought, and the cerebellar cognitive affective syndrome. Journal of Neuropsychiatry and Clinical Neurosciences 16, 367-378.

Scott JA, Schumann CM, Goodlin-Jones BL, Amaral DG (2009). A comprehensive volumetric analysis of the cerebellum in children and adolescents with autism spectrum disorder. Autism Research 2, 246-257.

Shaw P, Eckstrand K, Sharp W, Blumenthal J, Lerch JP, Greenstein D, Clasen L, Evans A, Giedd J, Rapoport JL (2007). Attention-deficit/hyperactivity disorder is characterized by a delay in cortical maturation. Proceedings of the National Academy of Sciences USA 104, 19649-19654.

Shaw P, Malek M, Watson B, Sharp W, Evans A, Greenstein D (2012). Development of cortical surface area and gyrification in attention-deficit/hyperactivity disorder. Biological Psychiatry 72, 191-197.

Shaw P, Sharp WS, Morrison M, Eckstrand K, Greenstein DK, Clasen LS, Evans AC, Rapoport JL (2009). Psychostimulant treatment and the developing cortex in attention deficit hyperactivity disorder. American Journal of Psychiatry 166, 58-63.

Simmons A, Westman E, Muehlboeck S, Mecocci P, Vellas B, Tsolaki M, Kloszewska I, Wahlund LO, Soininen H, Lovestone S, Evans A, Spenger C (2011). The

AddNeuroMed framework for multi-centre MRI assessment of Alzheimer's disease: experience from the first 24 months. International Journal of Geriatric Psychiatry 26, 75-82.

Simonoff E, Pickles A, Charman T, Chandler S, Loucas T, Baird G (2008). Psychiatric disorders in children with autism spectrum disorders: prevalence, comorbidity, and associated factors in a population-derived sample. Journal of the American Academy of Child and Adolescent Psychiatry 47, 921-929.
Stoodley CJ, Schmahmann JD (2009). Functional topography in the human cerebellum: a meta-analysis of neuroimaging studies. NeuroImage 44, 489-501.

Stoodley CJ, Schmahmann JD (2010). Evidence for topographic organization in the cerebellum of motor control versus cognitive and affective processing. Cortex 46 , 831-844.

Stoodley CJ, Valera EM, Schmahmann JD (2012). Functional topography of the cerebellum for motor and cognitive tasks: an fMRI study. NeuroImage 59, 1560-1570.

Tiemeier H, Lenroot RK, Greenstein DK, Tran L, Pierson R, Giedd JN (2010). Cerebellum development during childhood and adolescence: a longitudinal morphometric MRI study. Neurolmage 49, 63-70.

Toal F, Daly EM, Page L, Deeley Q, Hallahan B, Bloemen O, Cutter WJ, Brammer MJ, Curran S, Robertson D, Murphy C, Murphy KC, Murphy DG (2010). Clinical and anatomical heterogeneity in autistic spectrum disorder: a structural MRI study. Psychological Medicine 40, 1171-1181.

Valera EM, Faraone SV, Murray KE, Seidman LJ (2007). Meta-analysis of structural imaging findings in attention-deficit/hyperactivity disorder. Biological Psychiatry 61, 1361-1369.

Valera EM, Spencer RMC, Zeffiro TA, Makris N, Spencer TJ, Faraone SV, Biederman J, Seidman LJ (2010). Neural substrates of impaired sensorimotor timing in adult attention-deficit/hyperactivity disorder. Biological Psychiatry 68, 359-367.

Verhoeven J, Cock P, Lagae L., Sunaert S (2010). Neuroimaging of autism. Neuroradiology 52, 3-14.

Vloet TD, Gilsbach S, Neufang S, Fink GR, Herpertz-Dahlmann B, Konrad K (2010). Neural mechanisms of interference control and time discrimination in attention-deficit/hyperactivity disorder. Journal of the American Academy of Child and Adolescent Psychiatry 49, 356-367.

Webb SJ, Sparks BF, Friedman SD, Shaw DW, Giedd J, Dawson G, Dager SR (2009). Cerebellar vermal volumes and behavioral correlates in children with autism spectrum disorder. Psychiatry Research 172, 61-67.

Wechsler D (1999). Wechsler Abbreviated Scale of Intelligence. The Psychological Corporation: San Antonio, TX.

Whitwell JL (2009). Voxel-based morphometry: an automated technique for assessing structural changes in the brain. Journal of Neuroscience 29, 9661-9664.

WHO (1994). The ICD-10 Classification of Mental and Behavioural Disorders. Clinical Descriptions and Diagnostic Guidelines. World Health Organization: Geneva.

Wiener M, Turkeltaub P, Coslett HB (2010). The image of time: a voxel-wise meta-analysis. NeuroImage 49, 1728-1740.

Willcutt EG, Doyle AE, Nigg JT, Faraone SV, Pennington BF (2005). Validity of the executive function theory of attention-deficit/hyperactivity disorder: a meta-analytic review. Biological Psychiatry 57, 1336-1346.

Yassa MA, Stark CE (2009). A quantitative evaluation of cross-participant registration techniques for MRI studies of the medial temporal lobe. NeuroImage 44, 319-327. 